\title{
Hybrid polyMOF materials prepared by combining an organic polymer with a MOF and their application for solar thermal energy storage
}

\author{
Md. Hasan Zahir, *a Aasif Helal,*b and Abbas S. Hakeem ${ }^{b}$ \\ ${ }^{a}$ Center of Research Excellence in Renewable Energy (CoRE-RE), \\ ${ }^{b}$ Center of Research Excellence in Nanotechnology (CENT) \\ King Fahd University of Petroleum \& Minerals, (KFUPM), Dhahran 31261, Saudi Arabia
}
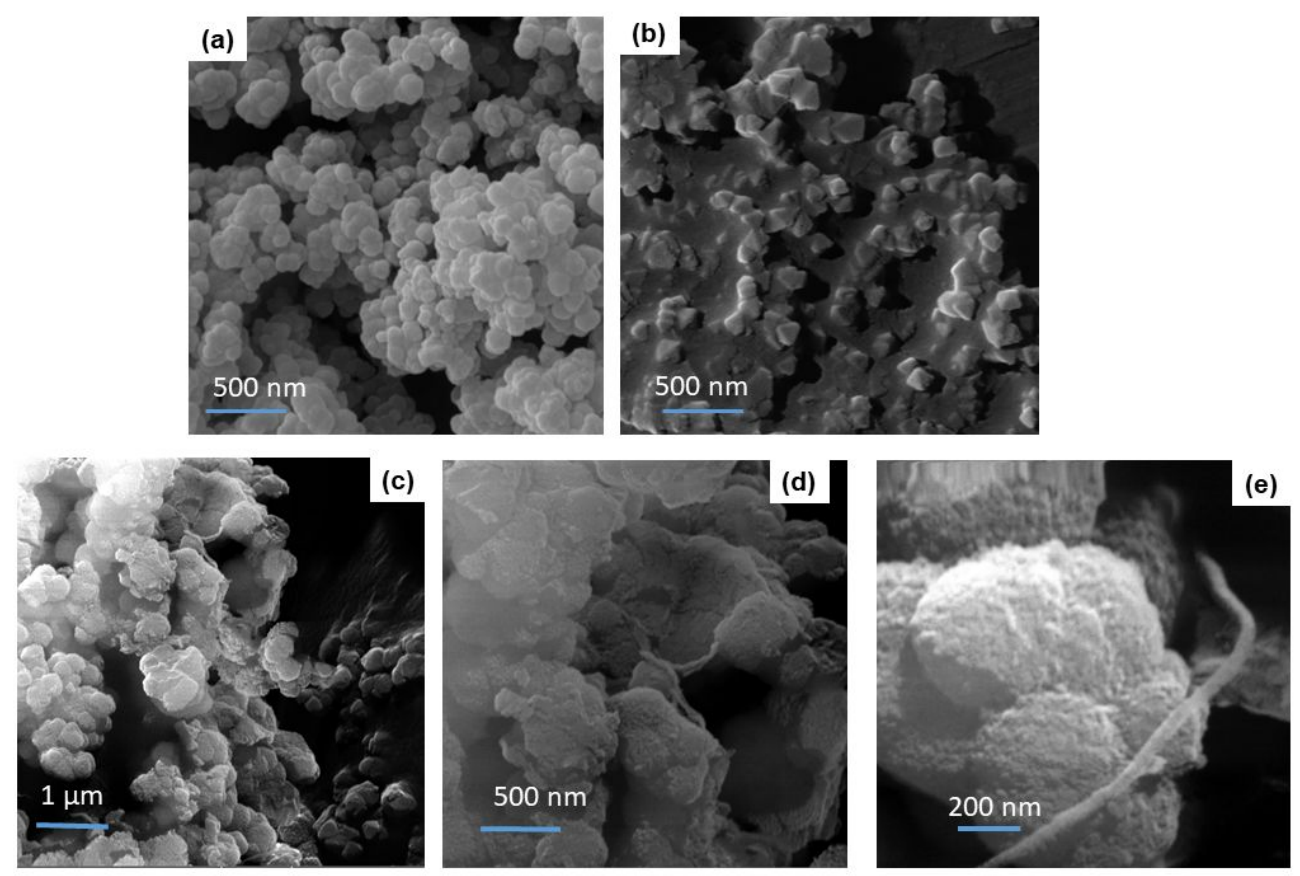

Fig. S1. FE-SEM images of the as-synthesized (a) UiO-66- $\mathrm{NH}_{2}$, (b) $\mathrm{PU}_{-} \mathrm{NH}_{2}-0.5$, and (c), (d), and (e) PU-0.5-CNT 5wt.\% at different magnifications. 